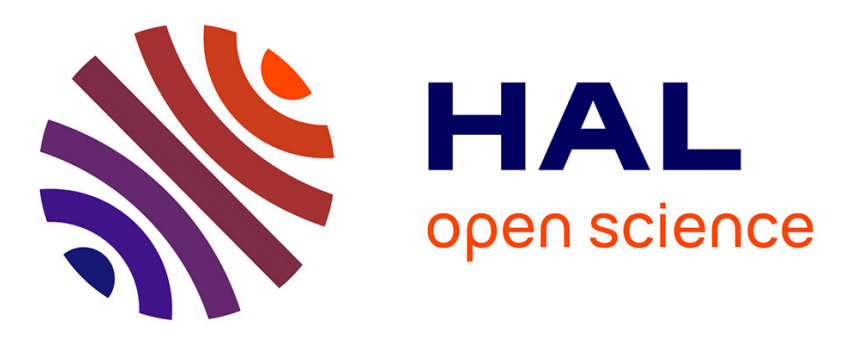

\title{
Contribution of polarimetric imaging for the characterization of fibrous surface properties at different scales.
}

\author{
Michel Tourlonias, Laurent Bigue, Marie-Ange Bueno
}

\section{To cite this version:}

Michel Tourlonias, Laurent Bigue, Marie-Ange Bueno. Contribution of polarimetric imaging for the characterization of fibrous surface properties at different scales.. Optics and Lasers in Engineering, 2010, 48, pp.75-82. 10.1016/j.optlaseng.2009.07.011 . hal-00849750

\section{HAL Id: hal-00849750 \\ https://hal.science/hal-00849750}

Submitted on 31 Jul 2013

HAL is a multi-disciplinary open access archive for the deposit and dissemination of scientific research documents, whether they are published or not. The documents may come from teaching and research institutions in France or abroad, or from public or private research centers.
L'archive ouverte pluridisciplinaire HAL, est destinée au dépôt et à la diffusion de documents scientifiques de niveau recherche, publiés ou non, émanant des établissements d'enseignement et de recherche français ou étrangers, des laboratoires publics ou privés. 


\title{
Contribution of polarimetric imaging for the characterization of fibrous surface properties at different scales.
}

\author{
Michel Tourlonias ${ }^{1,2}$, Laurent Bigué ${ }^{2}$, Marie-Ange Bueno ${ }^{1}$ \\ ${ }^{1}$ Laboratoire de Physique et Mécanique Textiles (CNRS-UMR 7189) \\ ENSISA-Werner - Université de Haute-Alsace - 11 rue Alfred Werner - 68093 Mulhouse, France \\ 2 Laboratoire Modélisation Intelligence Processus Systèmes (EA 2332) \\ ENSISA-Lumière - Université de Haute-Alsace - 12 rue des Frères Lumière - 68093 Mulhouse, France
}

\begin{abstract}
The point in using polarimetric imaging for surface characterization is highlighted in this paper. A method for the evaluation of nonwoven surface properties at microscopic and macroscopic scales is described. This method is based on a polarimetric apparatus and various image processing operations are then performed depending on the studied scale. Polarimetric imaging applied to nonwovens, particularly degree of polarization imaging, highlights texture inhomogeneities. At both scales, image processing techniques were designed to analyze surface zones of different textures. At the macroscopic scale, an basic image processing was developed in order to detect the nonwoven manufacturing process defects. Moreover at the microscopic scale, i.e. at the fiber scale, image processing was adapted to evaluate fiber orientation within nonwovens, which is known to be an important information for mechanical behavior prediction.
\end{abstract}

\section{Keywords}

polarimetric imaging, image processing, texture, nonwovens, fiber orientation.

\section{Introduction}

Considering a textile product, we can see that its mechanical properties are very important. Tension, compression, friction, bending, shear are key parameters which are essential in the future use of the product and are commonly studied [1]. We already developed techniques to evaluate the surfaces properties of textile fabrics [24]. In this paper, we improve these techniques and develop new ones for nonwoven characterization by the use of polarimetric imaging.

In the literature, using polarimetric properties of fibrous surfaces in order to characterize surface properties or fiber orientation in nonwovens was not reported until now. All the proposed methods use intensity snapshots of the surface which are post-processed. In this paper we highlight some surfaces properties of the fabrics (i.e. nonwoven in the following) thanks to polarization.

In this manuscript, tensile properties of calendered nonwovens are considered. They depend on elements at two scales of the surface: bonding points, i.e. macroscopic scale, and fiber orientation within the fibrous entanglement [5-7], i.e. microscopic scale. Whereas most authors classically take intensity snapshots they process with more or less complex image processing techniques [8-21], we rather intend to extract the relevant information with optical techniques before grabbing and implement 
subsequent data extraction from 2-D acquired signals. The latter data extraction often proves rather simple and therefore less subject to noise than classic image processing techniques reported in [8-21]. This approach can seem less flexible, since it requires the development of specific apparatus, but it allows us to extract information hardly visible in intensity images, which cannot be recovered by image post-processings [3, 4, 22].

Section 2 deals with a state of the art of nonwoven characterization, at both scales of interest. In Section 3 our apparatus and image processing techniques are described after a paragraph which reminds polarimetry basics. The following section presents our test samples and the results we obtained are finally reported in Section 5.

\section{Fabric characterization: state of the art}

A nonwoven fabric, generally simply called nonwoven, is a multiscale structure. Its basic component is fiber or filament, respectively with several centimeters to several kilometers length. Fiber or filament is about several tens of microns thick; that is the microscopic scale. These elements are entangled in a pseudo random way as a web. Actually fiber orientation is often slightly predominant along the machine direction. Then the cohesion between these fibers or filaments can be achieved by different processes: thermal (thermobonding), mechanical (needling or water or air jetting) or chemical process. Possible applications are for instance baby diapers, agriculture, surgical blouse for thermobonded nonwovens, sound insulation or civil engineering industry for needlemat, towelettes and sweeping tissues for water and air jet nonwovens, table napkin for the chemical nonwovens. In our study, we only experimented with thermobonded nonwovens.

\subsection{Macroscopic Scale}

By considering nonwoven surfaces or more generally textile surfaces, two kinds of defects can be found. These defects can be local defects or gradual changes of the manufacturing process of the surface.

Based on image processing several methods present the detection of local defects in textiles surfaces or other surfaces with a repetitive pattern. These method consist in removing the global repetitive pattern and this processing allows the user to highlight local defects.

Tsai et al. [8] use the Fourier transform of textile images and the shape (amplitude and frequency) of its peaks expresses possible defects of the periodical texture. Wood [9] also uses the Fourier transform in order to analyze the modifications of the state of surface of textile carpets through the modifications of their texture properties. Several filtering techniques are used in order to process these signals and for instance Tsai [10, 11] Shakher [12] or KreißI [13] use filtering methods based on wavelet transform. These processes allow the user to obtain images where only defects appear, without any periodical structure. These images are often compared to images of reference textile surfaces.

In our case, we are not interested in detecting local defects but the differentiation has to be done between samples whose characteristics have changed during the manufacturing process. 
Polarization could clearly improve existing techniques through improvement of the contrast of images and for our samples; in the literature the study would have to be realized on repetitive pattern which is up to now removed of the study and therefore not studied.

\subsection{Microscopic Scale}

Several methods based on image processing exist which allow the user to determine the fiber orientation. In their study of nonwoven fabrics, Hearle et al. [21] take inventory of different methods in order to determine fiber orientation. Studying transmitted light and directional phenomena of dichroism and birefringence does not seem to give good results. A tedious method based on direct visual method is reported to perform better. The angle between each fiber and a marker line, process direction for instance, is measured. Then the count of fibers in each $10^{\circ}$ angular interval is determined and each value is reported to the total number of fibers. Then results are presented under the guise of histograms. This manual process corresponds to the means available in 1963.

Pourdeyhimi et al. [14-19] realized a complete study. First, they modeled an image of nonwoven in order to apply their image processing on images whose properties are known. These simulated nonwovens consist of straight continuous filament webs. Pourdeyhimi et al. present several methods in order to determine the percentage of fibers in each direction. A tracking algorithm is tested on these known samples whereby each fiber can be tracked and the end-to-end chord of each fiber can be determined. This chord gives the orientation of the fiber. So it is possible to determine the orientation distribution in the textile surface. In another part of the study, fiber orientation is determined using digital Fourier transform of a snapshot. Tests are realized on the same simulated nonwovens. In the following part of the study, a flow field analysis is presented. It allows the user to determine the local orientation information. All these methods are compared and the latter is reported to be the most accurate but it only gives the mean orientation angle. Other methods give the orientation distribution functions and the tracking method is presented as the best method when considering off-line measurements. The last part of the series of papers presents the application of these methods to real webs. A contrast enhancement procedure is necessary before thresholding. The tracking method is presented as the best in order to determine the orientation distribution functions whereas Fourier transform proves better for quality control. A device is presented in order to realize an optical Fourier transform. Results obtained with both Fourier studies are similar and the main advantage of the optical method is speed. Pourdeyhimi et al. [20] also present a method based on Hough transform but this method seems to be complex and slow.

\section{Image acquisition}

\subsection{Polarimetry basics}

Whole polarimetric information about a monochromatic plane wave is all summarized in the Stokes Vector [23]: 


$$
\overrightarrow{\mathrm{S}}=\left[\begin{array}{l}
\mathrm{S}_{0} \\
\mathrm{~S}_{1} \\
\mathrm{~S}_{2} \\
\mathrm{~S}_{3}
\end{array}\right]=\left[\begin{array}{c}
\mathrm{I}_{0}+\mathrm{I}_{90} \\
\mathrm{I}_{0}-\mathrm{I}_{90} \\
\mathrm{I}_{+45}-\mathrm{I}_{-45} \\
\mathrm{I}_{\mathrm{r}}-\mathrm{I}_{\mathrm{l}}
\end{array}\right]
$$

where $\quad \mathrm{I}_{0}$ : linearly polarized component along horizontal axis

$l_{90}$ : linearly polarized component along vertical axis

$\mathrm{I}_{+45}$ : linearly polarized component at $45^{\circ}$

$\mathrm{I}_{-45}$ : linearly polarized component at $-45^{\circ}$

$\mathrm{I}_{\mathrm{r}}$ : right circularly polarized component

I,: left circularly polarized component

$\mathrm{s}_{0}$ describes the total intensity and the 3 others parameters correspond to polarimetric information of the wave. $s_{1}$ is about horizontal and vertical polarizations, the $\pm 45^{\circ}$ components are defined through $s_{2}$ and the right and left circular polarization are included in $s_{3}$ component. It is possible to establish the following relation between Stokes parameters:

$\mathrm{s}_{0}^{2} \geq \mathrm{s}_{1}^{2}+\mathrm{s}_{2}^{2}+\mathrm{s}_{3}^{2}$

and the degree of polarization of the wave:

$$
\mathrm{DOP}=\frac{\sqrt{\mathrm{s}_{1}^{2}+\mathrm{s}_{2}^{2}+\mathrm{s}_{3}^{2}}}{\mathrm{~s}_{0}}
$$

In the case where we only have at disposal two polarization crossed measurements, the partial degree of polarization can be defined [24, 25]:

$$
\mathrm{DOP}_{\text {partial }}=\frac{\mathrm{I}_{\alpha}-\mathrm{I}_{\alpha+\frac{\pi}{2}}}{\mathrm{I}_{\alpha}+\mathrm{I}_{\alpha+\frac{\pi}{2}}}
$$

If one of these directions corresponds to direction of the incident light, then $\alpha=0^{\circ}$ and the relation becomes:

$S=\frac{\left|I_{0}-I_{90}\right|}{I_{0}+I_{90}}$

where in summary $\mathrm{I}_{0}$ : linearly polarized component along vertical axis (aligned with incident polarization)

Igo: linearly polarized component along horizontal axis

Stokes polarimetry consists in measuring Stokes vector of a transmitted or reflected wave by an object [26-28].

\subsection{Description of the technique}

This study follows a previous study realized by the authors, during which a nonimaging optical texturometer was developed [3]. It allows the user to detect periodical structure elements of textile surfaces through the study of the light reflected by the sample. The same study realized on the degree of polarization of this reflected light highlights some phenomena. In order to better understand these phenomena, a fully polarimetric imaging device was implemented. Thus, an image in degree of polarization of the samples can be obtained [4].

A preliminary study on polypropylene nonwovens allowed us to determine that in spite of birefringent properties of polypropylene material, light reflection on these 
surfaces induces only depolarization phenomena at the macroscopic scale [29]. At this scale, there is no main reflection direction due to the fibrous disorder. Hence, this study can come down to evaluate the degree of polarization (DOP) of the light reflected by our samples under linear polarized illumination [24] as defined in equation 3.

To evaluate DOP, a polarizer is theoretically sufficient. Nevertheless, many polarizers exhibit parallelism issues between their two sides, that induces pixel shifts between the two images which are necessary for the calculation of the image in degree of polarization. To avoid the need for a digital image registration, a polarimetric imaging device based on the principle presented by Terrier et al. [30] was implemented. Thus, the whole Stokes vector (eq. 1) can be determined by rotating a N/4 plate.

Experimental considerations usually lead to use more than 4 angles of the $N / 4$ plate We use 5 angles: $-\frac{\pi}{4} ;-\frac{\pi}{8} ; 0 ; \frac{\pi}{8} ; \frac{\pi}{4}$. The symmetry between chosen angles simplifies calculation.

In this case, since the incident probe is polarized along the vertical axis, $I_{0}$ and $I_{90}$ are renamed respectively $\mathrm{I}_{/ /}$and $\mathrm{I}_{\text {cross. }}$.

As only depolarization phenomena took place (which was experimentally verified), the degree of polarization is reduced to:

$$
\mathrm{P}=\frac{\mathrm{I}_{\mathrm{pol}}}{\mathrm{I}_{\text {tot }}}=\frac{\sqrt{\mathrm{s}_{1}{ }^{2}}}{\mathrm{~s}_{0}}=\frac{\left|\mathrm{s}_{1}\right|}{\mathrm{s}_{0}}=\frac{\left|\mathrm{I}_{/ /}-\mathrm{I}_{\text {cross }}\right|}{\mathrm{I}_{/ /}+\mathrm{I}_{\text {cross }}}
$$

\subsection{Optical Device}

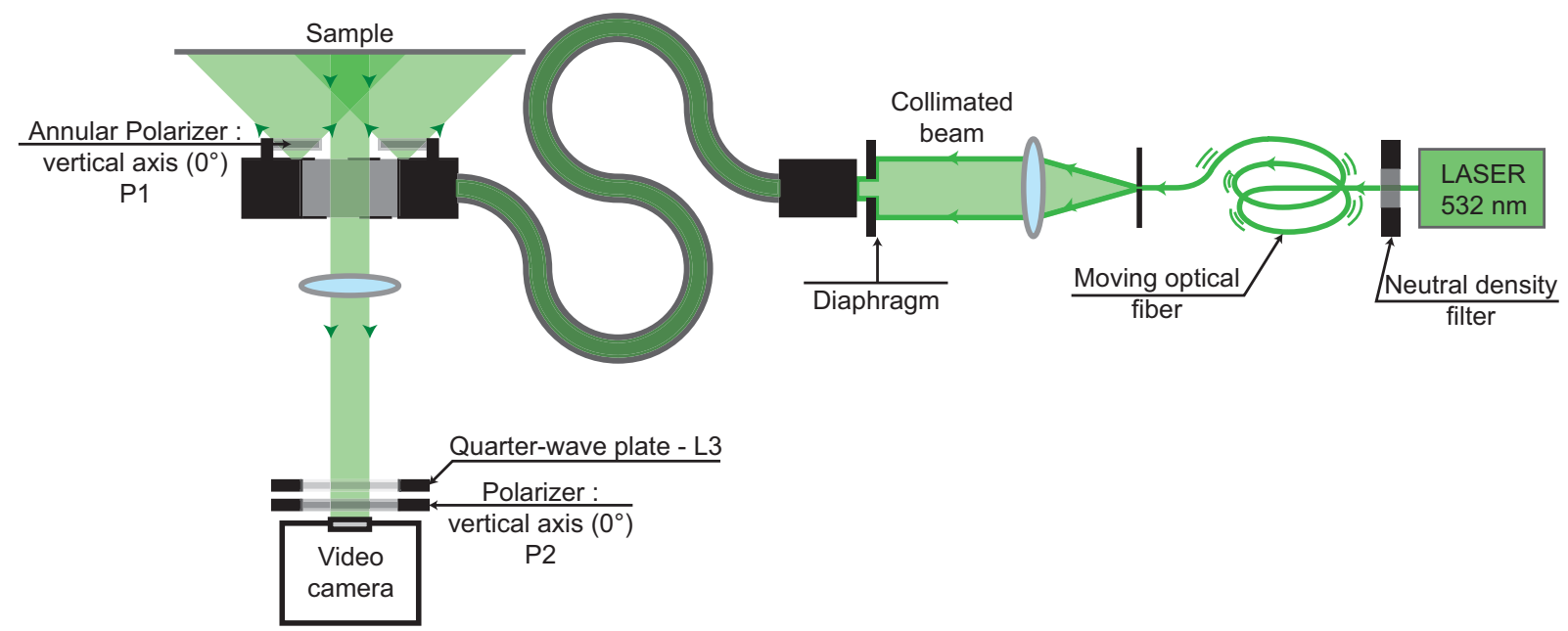

Fig. 1: Polarimetric imaging device

The optical assembly is shown in Fig. 1. The sample is lit by an annular system. The laser beam follows an optical fiber light guide and goes through an annular polarizer before reaching the sample. Therefore, the reflected beam reaches the camera directly without any deviation. The annular lighting device prevents us from using a beamsplitter cube which may modify polarization of the incident beam and which causes a $50 \%$ light loss when the beam goes through it. By rotating the quarterwave plate it is possible to acquire the images necessary to compute the 4 Stokes images according to Terrier's method [30]. In the present study, each image, for each of the 5 orientations of the quarter-wave plate, is an average of 3 successive acquisitions. 
From Stokes vector, it possible to determine the total light intensity of the reflected light by

$$
\mathrm{I}=\mathrm{I}_{/ /}+\mathrm{I}_{\text {cross }}
$$

During the study, the used intensity images are obtained with this formula in order to be in the same experimental conditions and the image processing is realized on the exactly identical zones of the samples.

The same set of acquired images allows the user to realize the two parts of the study about the characterization of the texture of the surfaces and about fiber orientation.

\section{Material}

Samples of polypropylene spunbonded nonwovens were used. The cohesion of the web of fibers is given by thermobonded points (Fig. 2). This process is called calendering. The web goes between two heated rollers. One of them is covered by dots and fibers are thermobonded at the contact between these dots and the other cylinder, under a given pressure and a given temperature.

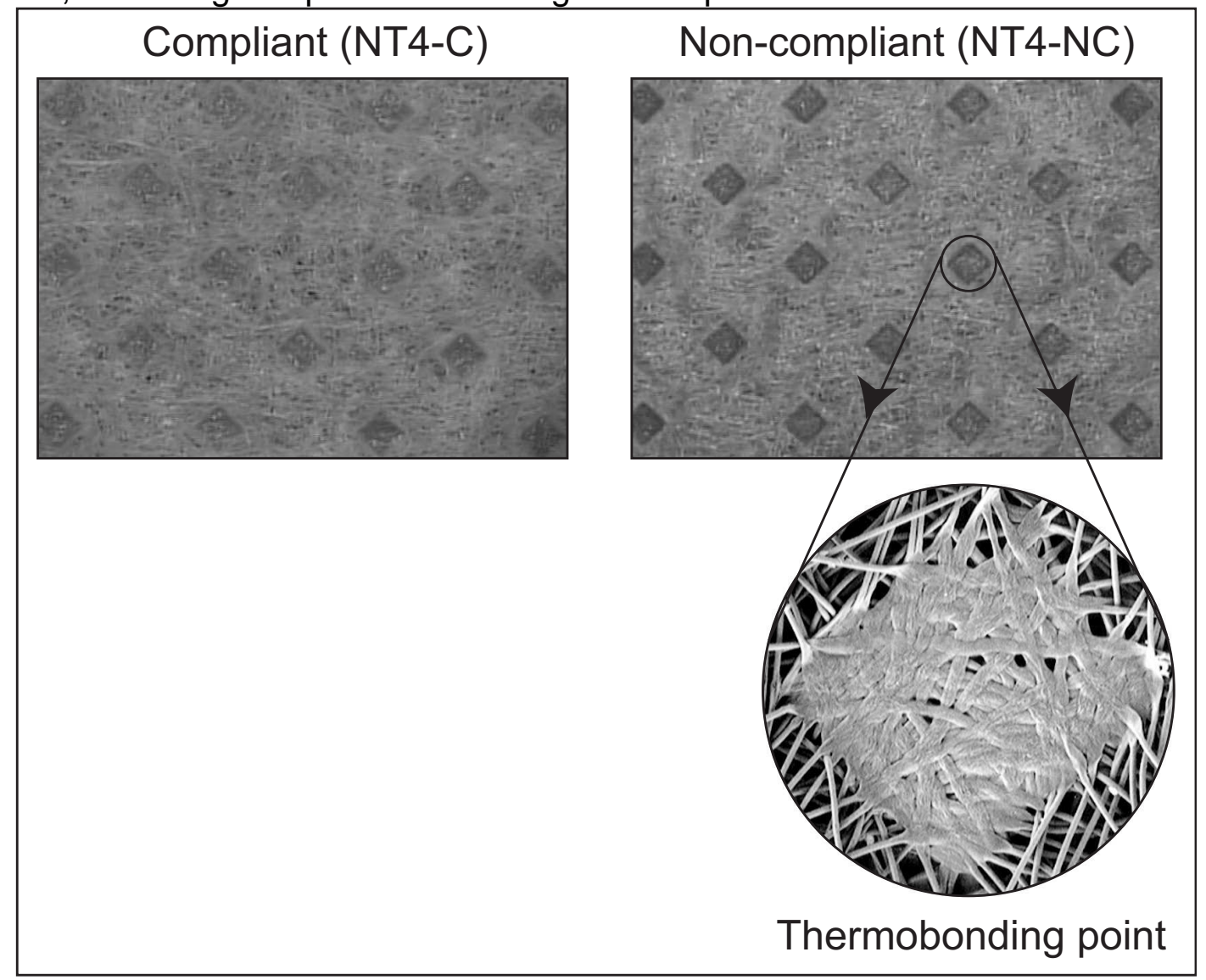

Fig. 2: Description of our nonwoven samples

Two versions of the same nonwoven are available. A trained sensorial panel evaluated their touch as being different. A sample, named NT4-C, is judged as meeting the manufacturer's requirements, therefore it is compliant. It is not the case for the other sample, which is named NT4-NC, thus it is non-compliant. Pictures of these nonwovens and description of their structure periodicities due to the calendering pattern are illustrated in Fig. 2. 


\section{Results and discussion}

\subsection{Characterization of the texture at macroscopic scale}

As explained above, a calendered nonwoven consists of two parts. The first one is the bonding points or zones and the second one is the fibrous background. The purpose in this part of the work consists in studying both nonwovens in these two zones thanks to polarimetric imaging. In Fig. 3 , we are presenting the different elementary zones that can be defined in these nonwovens. The analysis is realized by averaging values in the different zones and then by comparing results obtained with images in degree of polarization and images obtained in intensity. In particular, results obtained by averaging gray level values along the columns of the basic stripe are presented.

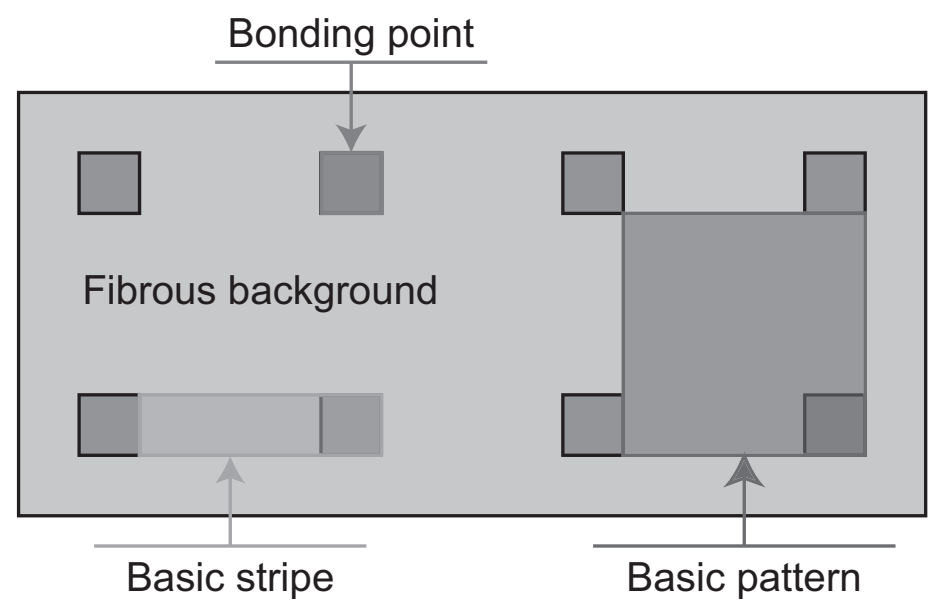

Fig. 3: Analysis zones of the spunbonded nonwovens

We can see that images in degree of polarization allow us to better differentiate the different zones of spunbonded nonwovens than intensity images.
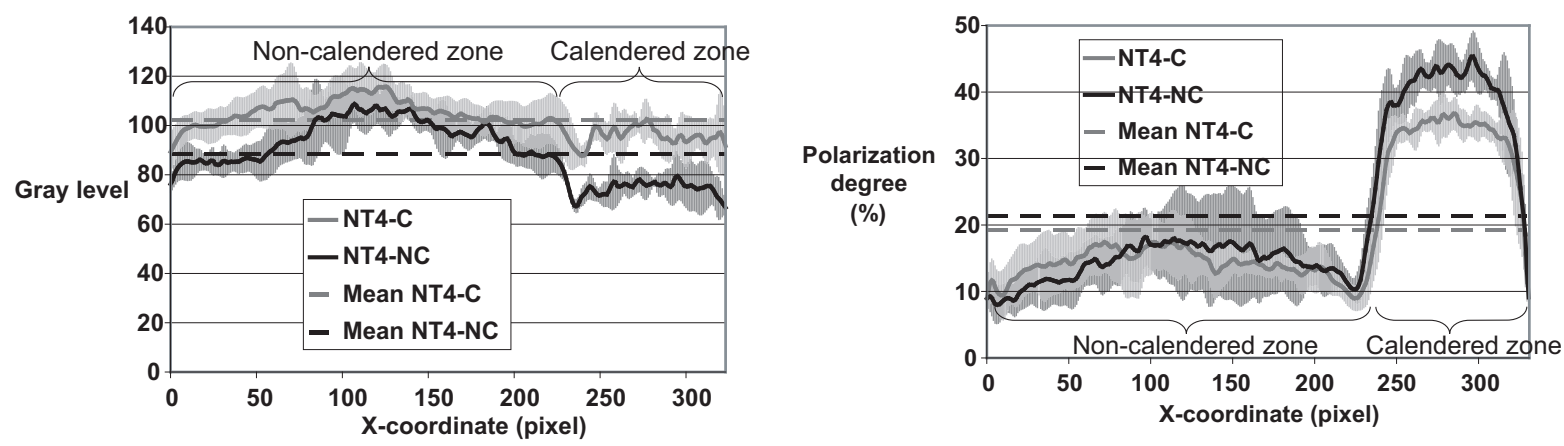

Fig. 4: Results obtained with polarimetric imaging of nonwovens. The curves are obtained by averaging gray level values along vertical direction. Results obtained with NT4 samples with images in intensity (a) and in degree of polarization (b)

Firstly, images in degree of polarization can differentiate much better the bonding points from the fibrous background than intensity images (Fig. 4a and 4b). Moreover, the difference that we can see in the diagram obtained by averaging values of each column is that images in degree of polarization permit to better differentiate the 
complying sample from the non-complying sample. It is obvious that the difference comes from the bonding point whereas the polarimetric properties of the fibrous background prove similar. On the graphs (Fig. $4 a$ and $4 \mathrm{~b}$ ) we added the standard deviation calculated along each column for the whole curves which highlights this tendency.

At the location where the fibers are melted during the calendering, the fiber disorder decreases (Fig. 2) and the bonding points tend to behave as mirrors, producing a specular reflection. This phenomenon does not modify the overall reflectance (intensity measures), but is highlighted when degree of polarization is considered.

For these two sets of samples the difference comes from the bonding points. Their ability to depolarize or not is directly related to the intensity of the calendering process. The degree of polarization of the bonding points for the non-complying sample is higher than that of the other one whereas figures obtained for the fibrous background are similar. It clearly means that the calendering process differs whereas in the beginning, both fibrous webs were identical. The pressure between the two rollers used in the process or the temperature of these rollers is probably higher than those in standard conditions.

\subsection{Characterization of the texture at microscopical scale}

The method presented here can be used for the fibrous background of the nonwoven located between the bonding points. This fibrous background is constituted of fibers whose orientation plays a major role in the mechanical properties of the surface and more particularly is directly linked to its tensile properties as mentioned for instance by Adanur et al. [5], Lin et al. [6] or Backer et al. [7]. In this study we use images in degree of polarization. This processing permits to highlight fibers parallel to the incident polarization direction.
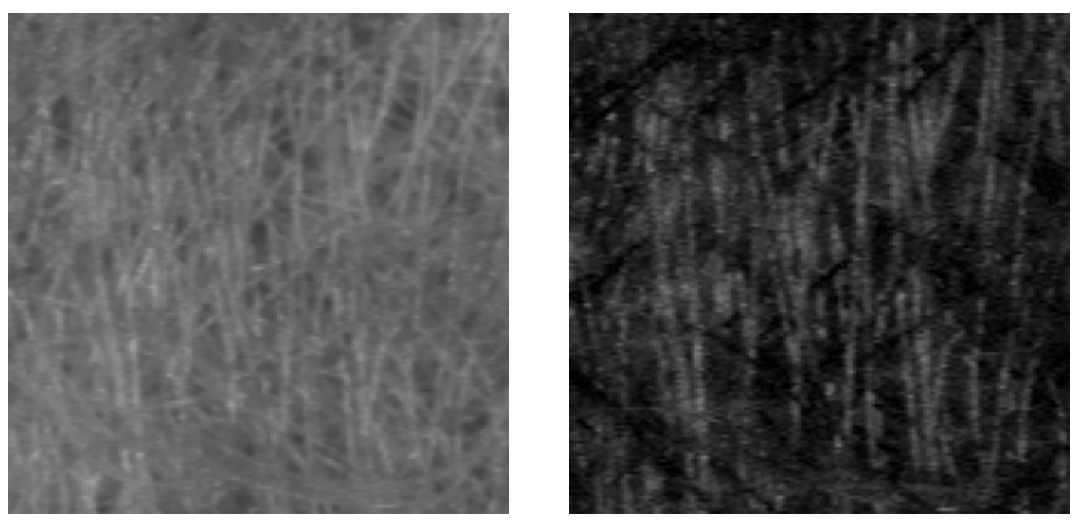

Fig. 5: Images of the fibrous background of a sample of NT4-NC in intensity (a) and in degree of polarization (b)

By studying images in degree of polarization, we clearly notice that the fibers are more visible (Fig. $5 \mathrm{a}$ and $5 \mathrm{~b}$ ), which allows us to implement rather basic processings of these images. Therefore, later on we will get interested in studying and determining the main orientation of these fibers from these images. The first step consists in selecting the circle tangential to the four closer bonding points. Then it is possible to select the square inscribed within this circle. A curve is obtained by 
averaging values in each column of the square for various orientations of the sample. The procedure was realized by mechanically rotating the sample over $360^{\circ}$. It is obvious that if fibers are parallel to the column of the square the obtained curves present some peaks, which corresponds to the presence or the absence of a fiber parallel to the column direction contrary to the case where the fibers are mainly crossed to these columns. By studying all directions of the sample it is possible to determine the main direction of the fibers (Fig. 6).

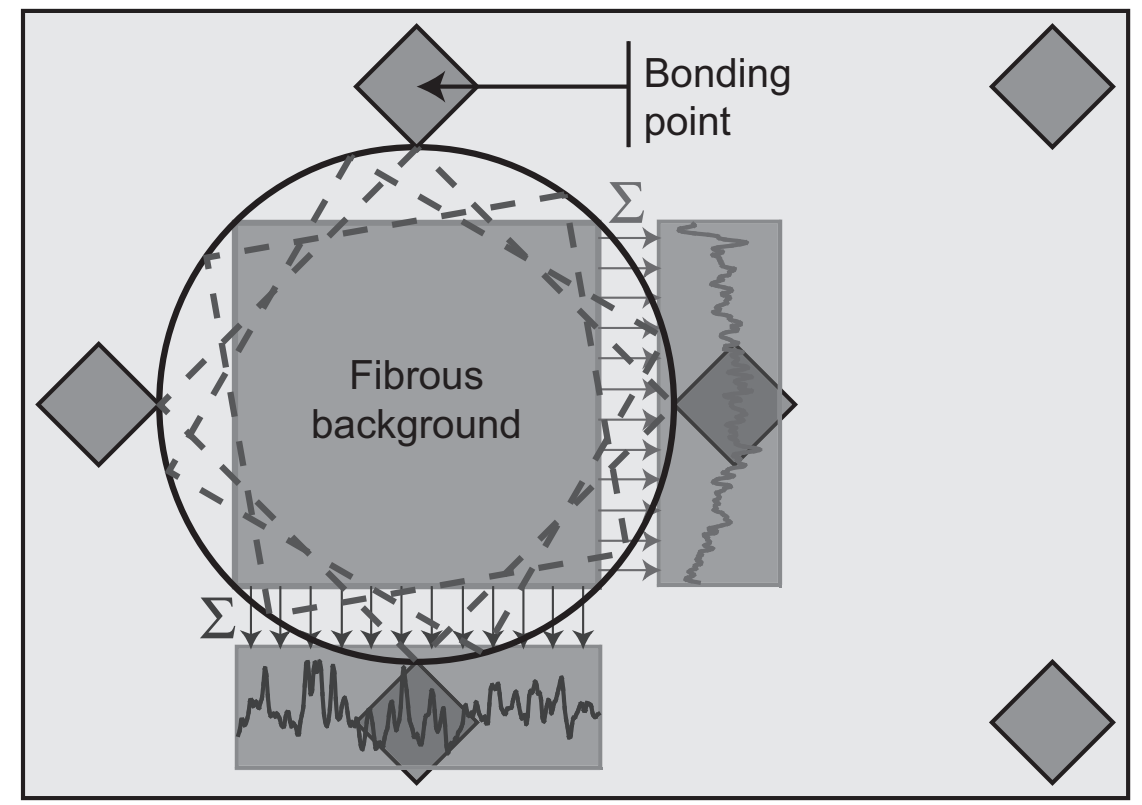

Fig. 6: Analysis zone in order to determine fiber orientation and example of obtained curves

In order to characterize the tendency of these curves which reveal fiber orientation, we used a calculation which is a statistical parameter, i.e. the mean slope of the curve. At the scale of the images, a fiber is approximately four pixels broad. So this slope is calculated with the following expression:

$\overline{\text { slope }}=\frac{1}{\mathrm{~L}-4} \sum_{\mathrm{i}=4}^{\mathrm{L}}\left|\mathrm{n}_{\mathrm{i}}-\mathrm{n}_{\mathrm{i}-4}\right|$

where $\quad L$ : width of the square (in number of pixels)

$\mathrm{n}_{\mathrm{i}}$ : average gray level of the $\mathrm{i}^{\mathrm{th}}$ column of the image

In order to complete the study of the spunbonded nonwoven NT4, we managed to determine the main orientation of the fibers of the surface. The technique described previously was applied. The image in degree of polarization of the surface is determined by a mechanical rotating the sample each $10^{\circ}$ from $0^{\circ}$ to $360^{\circ}$. It is not a numerical rotation that means there is no phenomenon of interpolation. As we define image in intensity with Stokes parameters, in each angular location we always analyze the exactly same part of the sample. That is why the comparison is directly possible. Examples of these curves are shown in Fig. 7. It is possible to see that curves in intensity are similar, whereas they differ in degree of polarization. The curve obtained by averaging gray level values at $0^{\circ}$ prese nt rather sharp peaks, whereas at $90^{\circ}$ the amplitudes of these peaks are smaller. 

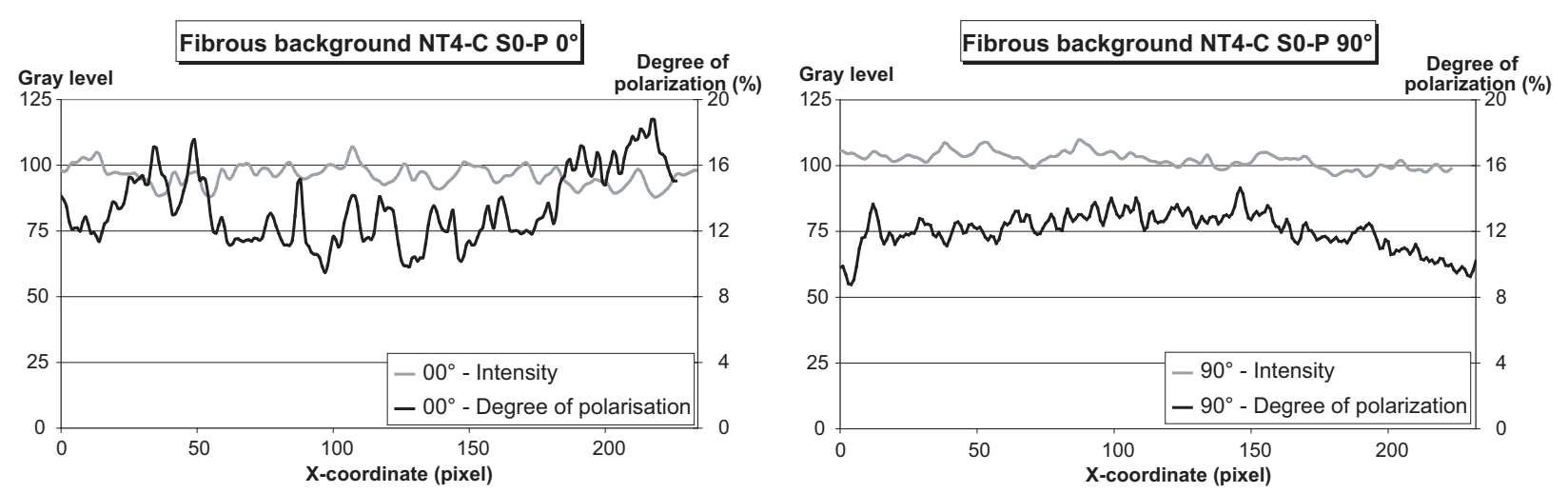

Fig. 7: Examples of curves obtained by averaging gray level values along the vertical direction of the images taken at two different angles $\left(0^{\circ}\right.$ and 909

As previously explained, the mean slope was calculated and it is possible to draw the graph reported in Fig. 8 through an angular representation as suggested by Hearle [21]. Obtained values were averaged from five images for each orientation of the sample and this graph presents the averages of these results. The same image processing was applied to image in intensity. Results are presented in Fig. 9. In order to get rid of light variations during the acquisition, each value has been normalized compared to the whole image intensity (eq. 9).

$\overline{\text { slope }}=\frac{1}{\overline{\mathrm{n}}(\mathrm{L}-4)} \sum_{\mathrm{i}=4}^{\mathrm{L}}\left|\mathrm{n}_{\mathrm{i}}-\mathrm{n}_{\mathrm{i}-4}\right|$

where $\quad \bar{n}$ : average gray level of the image

We can observe that these graphs are not exactly symmetrical. By rotating the samples we do not consider exactly the same zone of the sample and small differences appear.

The results show that the main orientation of the fiber corresponds to the direction $0^{\circ}-$ $180^{\circ}$ (Fig. 8 and 9). In this case this is the machi ne direction, i.e. the direction of the web during the manufacturing process. The tendency is the same for the two sets of samples whether they comply with the manufacturer's requirements or not. This tendency corresponds to values obtained by studying images in intensity. Nevertheless, considering DOP instead of intensity proves of interest because it provides a better discrimination: the ratio between figures obtained at $0^{\circ}$ and $90^{\circ}$, i.e. the directions in which the fibers are the most or the least oriented, is about 1.5 in intensity images and about 2 concerning images in degree of polarization.

Values obtained for NT4-C are lower than those obtained for NT4-NC. The tendency is the same all over the circular graph. This difference comes from a modification of the structure of the fibers due to the calendering process or during the manufacturing process of the fiber web which can lead to a modification of the birefringence as during a drawing process of such fibers or filaments [31]. These phenomena were already reported by Chand et al. [32]. Increased birefringence and crystallinity were measured in the bonded region as well as in the unbonded region. That is why the values obtained cannot be compared directly between two different samples but have to be normalized compared to other directions. For instance it is possible to compare the ratio between $0^{\circ}$ and $90^{\circ}$. 


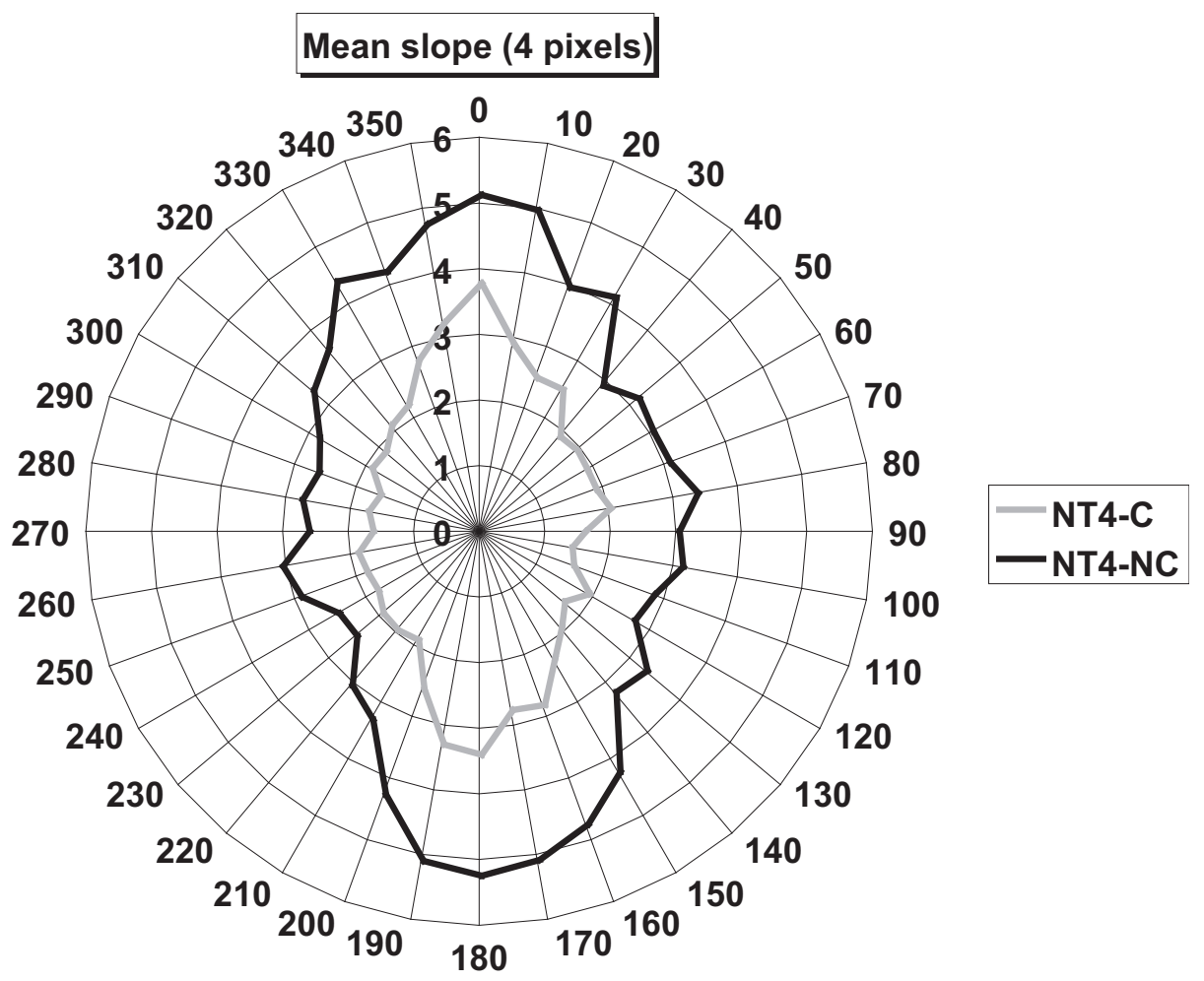

Fig. 8: Graph showing the main orientation of the fibers in the NT4 nonwovens from images in degree of polarization

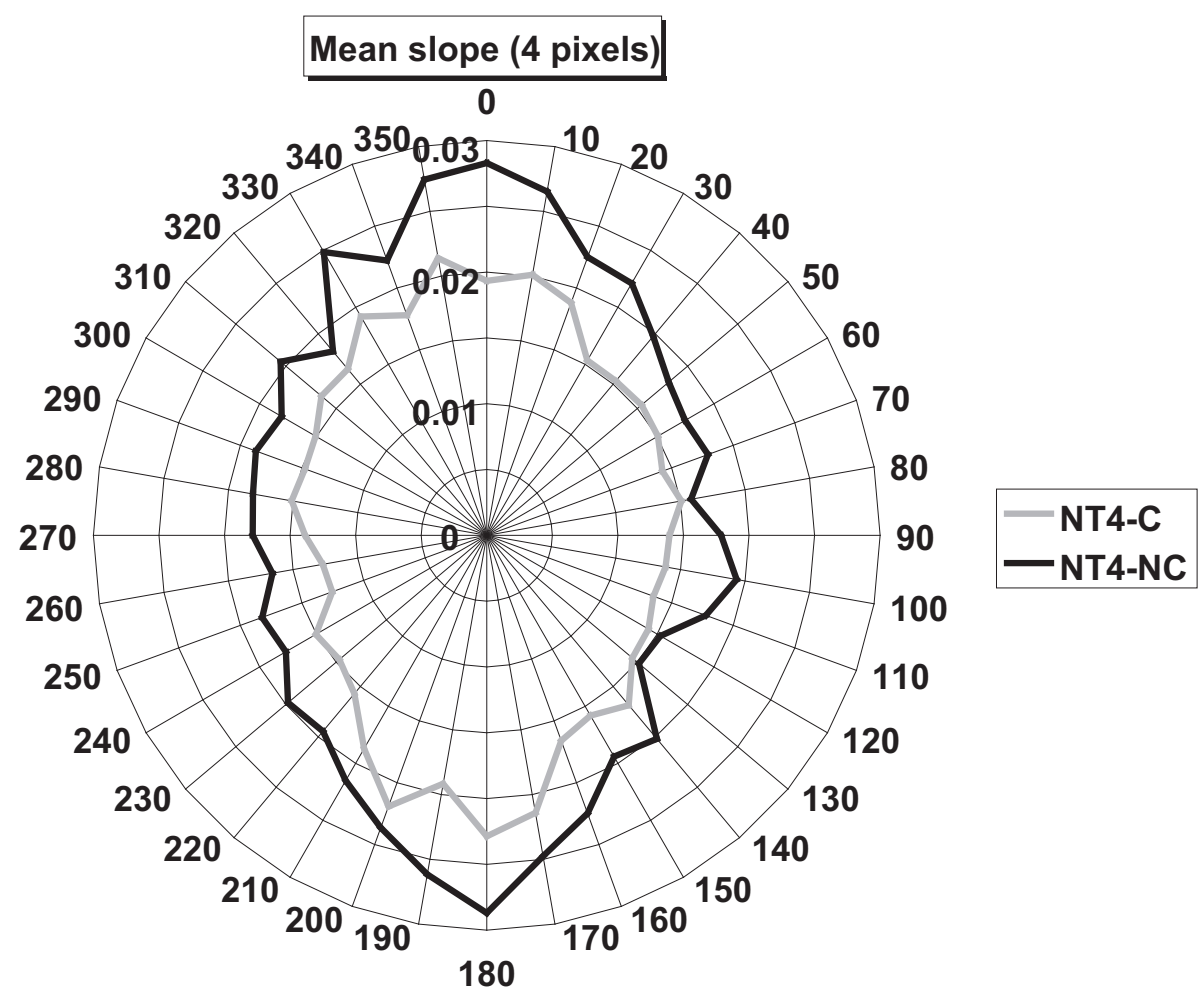

Fig. 9: Graph showing the main orientation of the fibers in the NT4 nonwovens from intensity images 


\section{Summary and perspectives}

This study allowed us to better characterize surface properties of nonwovens thanks to polarimetric imaging. First, using polarimetric imaging makes it possible to better determine zones (or regions) which have been calendered. The images in degree of polarization are also useful in order to determine a difference during the calendering process. When the intensity of the calendering increases, the depolarization phenomena decrease. Then the study of the fibrous background of the thermobonded nonwovens, along various directions, proves to be interesting in order to evaluate the main fiber orientation. The degree of polarization improves the determination of the main fiber orientation in the fibrous background of the nonwovens. It is also interesting to note that the image processing of the two parts of the study can be realized with the same optical device and with the same set of acquisitions.

We can imagine developing a system combining these two image processing techniques and then considering both microscopic and macroscopic scales. The first step will consist in differentiating the different zones of the sample thanks to the images in degree of polarization. In this way it will be possible to quantify calendering process and after this first step it is possible to select the fibrous background in order to characterize the main orientation of the fibers of the nonwoven. It is conceivable to automate the acquisition of the images necessary to determine the images in degree of polarization: up to now the rotations of the quarter-wave plate are performed manually, but a motorized stage or even a liquid crystal waveplate could improve our device's ease of use. It will also be interesting to realize in parallel a study of the tensile properties of this nonwoven in all directions in order to link obtained results of fibrous orientation and calendering properties. In this way, it could be possible to determine a model of this type of nonwovens and to predict its properties without any destructive test.

\section{Acknowledgments}

The authors wish to thank Patrick Bachschmidt for his help in the English version of this paper.

\section{References}

1. S. Kawabata, The standardization and analysis of hand evaluation, Technical report: The textile machinery society of Japan, Osaka, 1980.

2. M.-A. Bueno, B. Durand and M. Renner, Noncontact Measurements of Sanding and Raising Effects, Textile Research Journal 69(8) (1999) 570-575.

3. M.-A. Bueno, B. Durand and M. Renner, Optical characterization of the state of fabric surfaces, Optical Engineering 39(6) (2000) 1697-1703.

4. M. Tourlonias, L. Bigué and M.-A. Bueno, Polarimetric measurements of fabric surfaces, Optical Engineering 46(8) (2007) 083602. 
5. S. Adanur and T. Liao, Fiber Arrangement Characteristics and Their Effects on Nonwoven Tensile Behavior., Textile Research Journal 69(11) (1999) 816.

6. J.-H. Lin, Z.-H. Xu, C.-H. Lei and C.-W. Lou, Effect of Fiber Arrangement on the Mechanical Properties of Thermally Bonded Nonwoven Fabrics., Textile Research Journal 73(10) (2003) 917.

7. S. Backer and D. R. Petterson, Some Principles of Nonwoven Fabrics, Textile Research Journal 30(9) (1960) 704-711.

8. D.-M. Tsai and C.-Y. Hsieh, Automated surface inspection for directional textures, Image and Vision Computing 18(1) (1999) 49-62.

9. E.-J. Wood, Applying Fourier and Associated Transforms to Pattern Characterization in Textiles, Textile Research Journal 60(4) (1990) 212-220.

10. D.-M. Tsai and B. Hsiao, Automatic surface inspection using wavelet reconstruction, Pattern Recognition 34(6) (2001) 1285-1305.

11. D.-M. Tsai and C.-H. Chiang, Automatic band selection for wavelet reconstruction in the application of defect detection, Image and Vision Computing $21(5)(2003) 413-431$.

12. C. Shakher, S.-M. Istiaque and S.-K. Singh, Application of wavelet transform in characterization of fabric texture, Proc. SPIE 4929 : Optical Information Processing Technology (2002) 158-164.

13. M. KreißI, S. Heiko and S. Teiwes, Optical processor for real-time detection of defects in textile webs, Proc. SPIE 3073 : Optical Pattern Recognition VIII (1997) 307-311.

14. B. Pourdeyhimi, Assessing Fiber Orientation In Nonwoven Fabrics, INDA J. Nonwoven Res 5(4) (1993) 29-36.

15. B. Pourdeyhimi, R. Ramanathan and R. Dent, Measuring Fiber Orientation in Nonwovens - Part I: Simulation, Textile Research Journal 66(11) (1996) 713-722.

16. B. Pourdeyhimi, R. Ramanathan and R. Dent, Measuring Fiber Orientation in Nonwovens - Part II: Direct Tracking, Textile Research Journal 66(12) (1996) 747753.

17. B. Pourdeyhimi, R. Dent and H. Davis, Measuring Fiber Orientation in Nonwovens - Part III: Fourier Transform, Textile Research Journal 67(2) (1997) 143151.

18. B. Pourdeyhimi and R. Dent, Measuring Fiber Orientation in Nonwovens - Part IV: Flow Field Analysis, Textile Research Journal 67(3) (1997) 181-187.

19. B. Pourdeyhimi, R. Dent, A. Jerbi, S. Tanaka and A. Deshpande, Measuring Fiber Orientation in Nonwovens - Part V: Real Webs, Textile Research Journal 63(3) (1999) 185-192. 
20. B. Pourdeyhimi and H. S. Kim, Measuring Fiber Orientation in Nonwovens: The Hough Transform., Textile Research Journal 72(9) (2002) 803.

21. J. W. S. Hearle and P. J. Stevenson, Nonwoven Fabric Studies - Part III: The Anisotropy of Nonwoven Fabrics, Textile Research Journal 33(11) (1963) 877-888.

22. A. Anand, V. K. Chhaniwal and C. S. Narayanamurthy, Hairiness measurement of textile yarns using crossed polarizers, Review of Scientific Instruments 76(7) (2005) 076104-3.

23. D. Goldstein, Polarized Light - Second Edition, revised and expanded, Marcel Dekker, New-York, Basel, 2003.

24. A. Al-Qasimi, O. Korotkova, D. James and E. Wolf, Definitions of the degree of polarization of a light beam, Optics Letters 32(9) (2007) 1015-1016.

25. P. Réfrégier, F. Goudail and N. Roux, Estimation of the degree of polarization in active coherent imagery by using the natural representation, Journal of The Optical Society of America A 21(2) (2004) 2292-2300.

26. J. E. Solomon, Polarization imaging, Appl. Opt.

Applied Optics 20(9) (1981) 1537-1544.

27. J. S. Tyo, D. L. Goldstein, D. B. Chenault and J. A. Shaw, Review of passive imaging polarimetry for remote sensing applications, Appl. Opt.

Applied Optics 45(22) (2006) 5453-5469.

28. F. A. Sadjadi and C. S. L. Chun, Remote sensing using passive infrared Stokes parameters, Optical Engineering 43(10) (2004) 2283-2291.

29. M. Tourlonias, Ph.D thesis: Caractérisation optique de surfaces textiles : aspects dynamiques et polarimétriques. 2005, Université de Haute-Alsace: Mulhouse. p. 117.

30. P. Terrier and V. Devlaminck, Système polarimétrique pour l'analyse d'images, Traitement du Signal 17(5-6) (2000) 479-490.

31. A. A. Hamza, A. E. Belal, T. Z. N. Sokkar, M. A. El-Bakary and K. M. Yassien, Measurement of the spectral dispersion curves of low birefringence polymer fibres, Optics and Lasers in Engineering 45(9) (2007) 922-928.

32. S. Chand, G. S. Bhat, J. E. Spruiell and S. Malkan, Structure and properties of polypropylene fibers during thermal bonding, Thermochimica Acta 367-368((2001) 155-160. 